\title{
tomviz: Providing Advanced Electron Tomography by Streamlining Alignment, Reconstruction, and 3D Visualization
}

Yi Jiang ${ }^{1}$, Elliot Padgett ${ }^{2}$, Marcus D. Hanwell ${ }^{3}$, Cory Quammen ${ }^{3}$, Chris Harris ${ }^{3}$, Shawn Waldon ${ }^{3}$, David A. Muller ${ }^{2,4}$, Robert Hovden ${ }^{5}$

1. Department of Physics, Cornell University, Ithaca, NY, USA

2. School of Applied \& Engineering Physics, Cornell University, Ithaca, NY, USA

${ }^{3 .}$ Kitware, Inc., Clifton Park, NY, USA

4. Kavli Institute at Cornell for Nanoscale Science, Ithaca, NY, USA

${ }^{5 .}$ Department of Materials Science and Engineering, University of Michigan, Ann Arbor, MI, USA

Electron tomography (ET) is widely used for 3D characterization of biological and material systems at nano-scales and meso-scales [1,2]. The success of an ET experiment critically depends on many image processing steps including tilt series alignment, tomographic reconstruction, segmentation, and visualization. Because each step demands unique algorithms developed by researchers from different fields of expertise, electron microscopists must piece together multiple software tools - making the workflow inefficient and non-reproducible. Here, we present the first full release of tomviz, v.1.0, an advanced and open platform that achieves high-throughput ET by providing state of the art functionality from basic image filters to 3D segmentation.

tomviz offers a broad collection of reconstruction methods, from traditional weighted back projection (favored by biological applications) to more advanced optimization-based techniques such as the totalvariation minimization method [3] that utilizes the concept of compressed sensing. To lower the barrier of complicated algorithms that involve multiple steps and parameters, the software provides live updates and image inspection tools that facilitate parameter selection and convergence test.

In addition to reconstruction, users can perform 2D tilt series processing such as background subtraction and noise filtering. With automatic alignment methods for image translation and tilt axis, it's possible to obtain reasonable reconstruction from raw data with a single click. Manual alignments are also supported with intuitive and flexible user interface. All data transforms are tracked step-by-step in a pipeline and can be shared among different computers.

For large 3D data sets, tomviz performs fast and interactive visualization and segmentation by combining the popular Insight Toolkit (ITK) and the Visualization Toolkit (VTK) [4]. The platform offers a variety of GPU-accelerated rendering modes beyond traditional 3D volumetric rendering by including adjustable lighting and maximum intensity projections. Many common morphology transformation and segmentation functions are also included. With more than 100 color maps available and tools for editing axis labels, users can easily to generate publication-quality figures and animations.

tomviz is open source and available for Windows, Mac OS X, and Linux. It is designed for intuitive graphical use by scientists, and built in $\mathrm{C}++$ and Python (with built-in Numpy, Scipy, FFTW and wrapped ITK/VTK). The package comes with sample ET data sets [5] and flexible plug-in capabilities for users to implement their own algorithms and applications, making the software a valuable resource for learning electron tomography and testing new techniques. The software has an active user base with over 2,500 downloads and a multitude of electron microscopists participating in its development. 
References:

[1] De Rosier, D. and Klug, A. Nature 217 (1968), p.130-134.

[2] Midgley, P.A. et al, Chemical Communications 10 (2001), p.907-908.

[3] E. Sidky et al, Physics in medicine and biology 53 (2008), p.4777-4807.

[4] M.D. Hanwell et al, SoftwareX 1-2, 9-12 (2015).

[5] B. Levin et al, Scientific Data 3 (2016).

[6] tomviz is supported from DOE Office of Science contract DE-SC0011385.

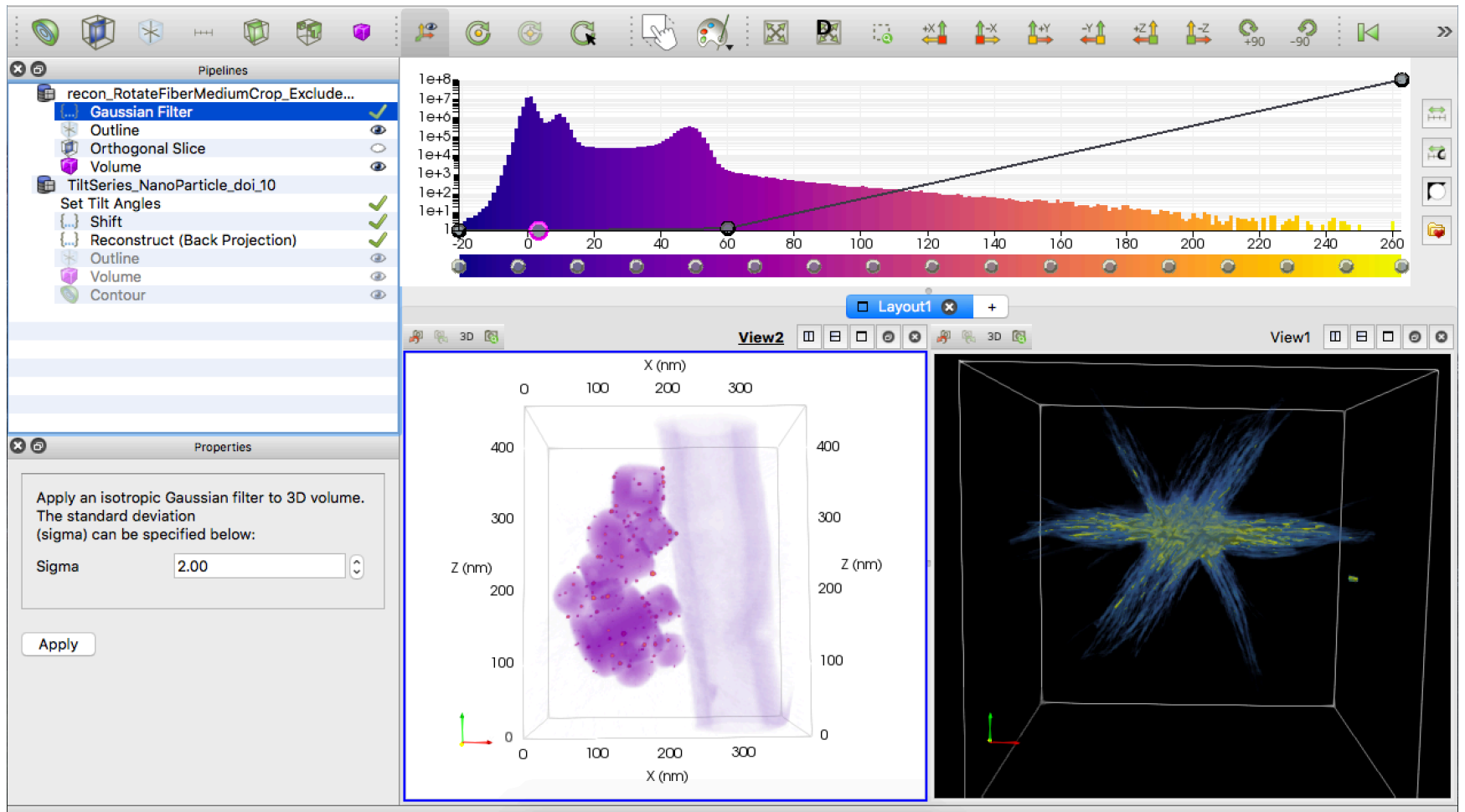

Figure 1. Graphical interface of tomviz 1.0. Multiple 3D objects can be visualized in different rendering modes at the same time. All data transforms are presented in the pipeline can be accessed and modified.
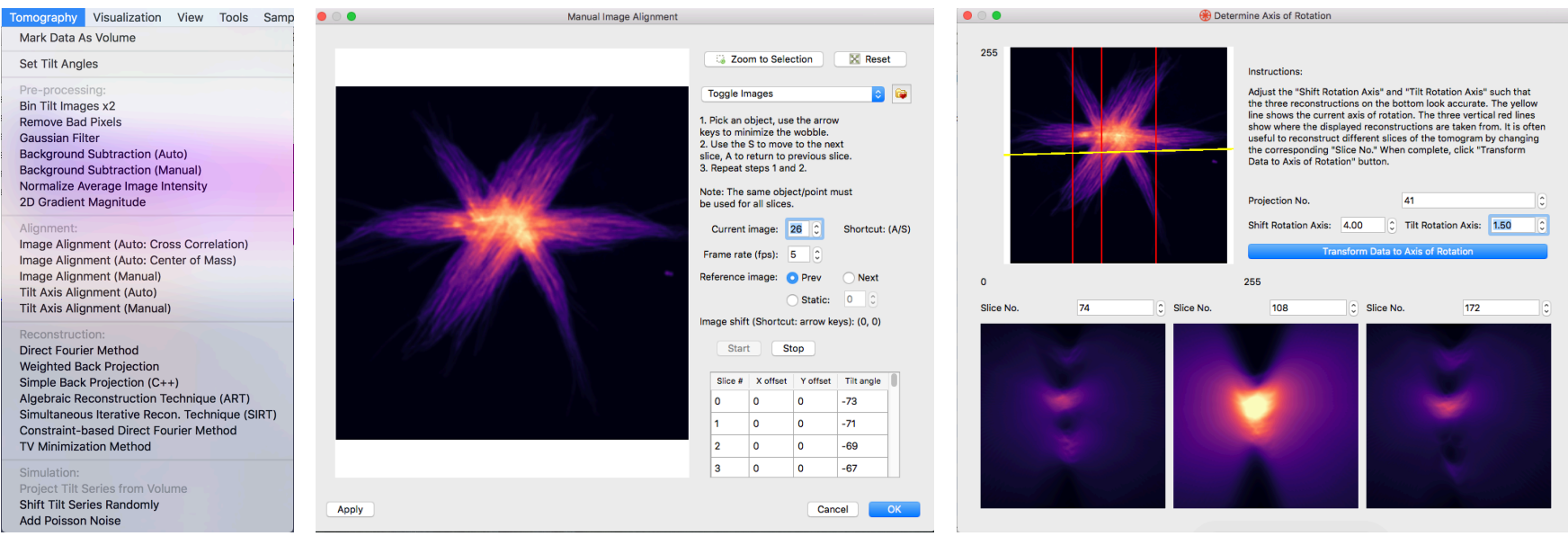

Figure 2. tomviz provides essential tools (left) for high-throughput electron tomography. Manual translation (middle) alignment and tilt axis (right) alignment come with intuitive and flexible UI. A broad collection of reconstruction techniques is included in the software. 